
\title{
R Reserach S Suare \\ The Effect of Necroptosis-related Gene Signature on the Progression, Prognosis, and Immune Microenvironment of Low-grade Glioma
}

\section{Kuo Zeng}

Renmin Hospital of Wuhan University

Guo Zhang

Renmin Hospital of Wuhan University

Wei Huang

Wuhan Cencer Hospital

Jianping Zhang

Liuzhou People's Hospital

Zhibiao Chen ( $\nabla$ chzbiao@126.com )

Renmin Hospital of Wuhan University

\section{Research Article}

Keywords: necroptosis, prognosis, low-grade glioma, immune, signature

Posted Date: December 13th, 2021

DOI: https://doi.org/10.21203/rs.3.rs-1156900/v1

License: (c) (1) This work is licensed under a Creative Commons Attribution 4.0 International License. Read Full License 


\section{Abstract}

\section{Background}

Despite the incorporation of various clinical and molecular criteria in the diagnosis and prognosis prediction of low-grade glioma, individual variation and risk stratification have not been completely explored. Necroptosis is considered closely related to different types of cancers, including low-grade gliomas. In this study, we obtained the necroptosis genes from the Kyoto Encyclopedia of Genes and Genomes website, extracted necroptosis genes from The Cancer Genome Atlas, and established a necroptosis-related gene signature (NECSig) through hazard analyses. Then we established a prognostic risk model consisting of four NECSig (BID, H2AFY2, MAPK9, and TNFRSF10B).

Result

Based on the model, the high-risk group is significantly associated with poorer overall survival. The accuracy of this model is further supported by the receiver operating characteristic curve. Then, we constructed a prognostic nomogram combining NECSig and clinical features, which shows good predictive power for stratification of survival risk. We discovered variations in the kind of immune infiltration, immune cells, and functions between the high-risk and low-risk groups using this risk model. We also showed that drug therapy is more sensitive in high-risk populations.

Conclusion

The results revealed a prognostic indicator of NECSig, which may provide information for immunological research and treatment of low-grade gliomas.

\section{Introduction}

Diffuse glioma is the most common type in terms of malignant brain tumor [1]. Glioblastoma, or grade IV glioma, is the most aggressive kind, with a five-year overall survival (OS) rate slightly around $5 \%$ [2]. Meanwhile, low-grade glioma (LGG) is generally a less aggressive tumor with a median survival rate of 510 years $[3,4]$. Total tumor excision, postoperative radiation, chemotherapy, and other comprehensive therapies are among the traditional therapeutic options. In recent years, many studies focused on integrating clinicopathological factors as prognostic biomarkers. However, a comprehensive knowledge of the pathomechanism and a more defined prognostic model remain critical for improving the overall survival and quality of life in LGG patients.

Necroptosis, which depends mainly on the activity of receptor-interacting protein 1 (RIPK1) and receptorinteracting protein 3 (RIPK3) kinases, is a type of cell death that occurs in the absence of apoptosis $[5,6]$. The death signal induces the activation of RIPK3 kinase, phosphorylates the specific executive protein mixed lineage kinase domain-like protein (MLKL) for cell necroptosis [6, 7]. Phosphorylated MLKL may then be controlled by oligomerization and translocates to the cell membrane structure, resulting in the 
degradation of cell membranes and organelle membranes, cell death, and intracellular material leakage [8-10].

In recent years, the signaling pathway of necroptosis has been widely studied. It has some common upstream signal elements with apoptosis, among which tumor necrosis factor receptor 1 (TNFR1) is the most deeply studied $[11,12]$. TNFa binds to TNFR1 on the plasma membrane, causing the TNF receptorassociated death domain (TRADD) to signal RIPK1 and recruit RIPK3 to form a necrosome [13]. Caspase8 is a key inhibitor of necroptosis as it may cleave and inactivate RIPK1 and RIPK3 [14]. If caspase-8 is inhibited, RIPK1 and RIPK3 interact with the RIP isotype domain, triggering necroptosis. This results in a cascade of automated and cross-phosphorylation of RIPK1 and RIPK3 [15-17]. Studies have demonstrated that some drugs are able to induce necroptosis in glioma cells through multiple molecular mechanics [18-20]. However, there is still no complete understanding of the mechanics and prognostic roles of necroptosis in glioma.

In this work, we built a prognostic model based on necroptosis-related mRNA features (including BID, H2AFY2, MAPK9, and TNFRSF10B), and comprehensively examined the relationship between the model and the prognostic features of LGG patients. The model has strong predictive performance, according to survival analysis and the receiver operating characteristic (ROC) curve. Multivariate Cox and stratified analyses suggest that this necroptosis-related gene signature (NECSig) feature acts as an independent prognostic factor of OS. To estimate the survival rate of these individuals, we created a nomogram that combined NECSig features with clinical parameters. The immunological statuses of the high-risk and low-risk group, as well as their responses to immunotherapy were compared. This four-gene-NECSigbased risk profile might be used to predict the prognosis and immunotherapy response of LGG patients.

\section{Results}

\section{Identification of NECDEs and Functional Enrichment Analysis}

Among 161 necroptosis-related genes extracted, a total of 12 genes were upregulated and 13 genes were downregulated when comparing the tumor tissues and normal tissues according to the following criteria: false discovery rate (FDR) $<0.05$ and $\mid \log 2$ (fold change) $\mid>1$ (Figure 1A, Table S2). The box plot depicts the expression levels of each differential gene in normal and malignant tissues (Figure 1B). We then performed GO and KEGG enrichment analyses. As for biological process enrichment, the NECDEs are significantly involved in extrinsic apoptotic-signaling pathway, regulation of cytokine-mediated signaling pathway, regulation of response to cytokine stimulus, regulation of cysteine-type endopeptidase activity involved in apoptotic process, programmed necrotic cell death, necrotic cell death, activation of cysteinetype endopeptidase activity involved in apoptotic process, positive regulation of cysteine-type endopeptidase activity involved in apoptotic process, positive regulation of cysteine-type endopeptidase activity, and necroptotic process (Figure 1C). The top 10 KEGG pathways from enrichment analysis of the NECDEs were necroptosis, influenza A, lipid and atherosclerosis, tuberculosis, measles, Hepatitis B, 
Coronavirus disease - COVID-19, toll-like receptor signaling pathway, NOD-like receptor signaling pathway, and Kaposi sarcoma-associated herpesvirus infection (Figure 1D).

(A) The NECDEs between LGG patients and normal controls. (B) The expression of different NECDEs in the normal group and tumor group. (C) The GO function enrichment analysis, including the top 10 pathways corresponding to biological processes, cellular components, and molecular functions. (D) The top 30 pathways in the KEGG functional enrichment analysis. $*, p<0.05 ; * \star, p<0.01 ; * \star \star, p<0.001$.

\section{Identification of a Prognostic NECSig}

The expression levels of 10 genes were then examined for connection with patient prognosis using univariate Cox regression. These values were discovered to have predictive significance for $L G G(p<0.05$; Figure 2A). Among them, six genes are potentially poor prognostic genes (PYGL, TNFRSF10B, IFNAR2, CASP1, IL1A, and TLR3), and four genes are potentially protective genes (BID, MAPK9, H2AFY2, and HIST3H2A). A total of four necroptosis-related mRNAs (BID, H2AFY2, MAPK9, and TNFRSF10B) were identified in a multivariate Cox proportional hazards regression analysis and were used to create a prognosis model based on NECSig. The estimated risk was calculated using the following formula: risk score $=(-0.073 \times$ expression level of BID $)+(-0.047 \times$ expression level of H2AFY2 $)+(-0.071 \times$ expression level of MAPK9) $+(0.089 \times$ expression level of TNFRSF10B $)$.

With the median risk score as the cutoff, there was a clear distinction between the high-risk and low-risk groups (Figure 2B). Each sample survival status is shown via a scatterplot (Figure 2C). The heat map shows how the NECSig gene is expressed in each sample (Figure 2D). A notable difference in OS was detected between the high-risk and low-risk groups. High-risk patients have a much lower survival rate than low-risk individuals ( $p<0.001$; Figure 2E). Furthermore, the findings show that the model has excellent sensitivity and specificity in LGG survival prediction using area under the curves (AUC). of 0.839 for one year, 0.815 for two years, 0.835 for three years, 0.688 for five years, 0.713 for seven years, and 0.784 for nine years (Figure 2F).

(A) The prognostic value of the 10 chosen NECDEs. (B) The risk curve is determined by the risk score for each sample. (C) The patient survival status of two groups. Blue and orange scatters indicate alive and dead respectively. (D) The distribution of four NECSig genes in the high-risk and low-risk groups based on the TCGA-LGG cohort. (E) Kaplan-Meier curves for patients in the high-risk and low-risk groups overall survival. (F) ROC curves of the risk score predicting one-, two-, three-, five-, seven-, and nine-year survival based on the TCGA-LGG cohort. AUC: area under the curve.

\section{Evaluation of NECSig as an independent prognostic factor for LGG}

We computed the hazard ratios (HRs) $(95 \% \mathrm{Cl})$ for the risk score to see if NECSig operates as an independent prognostic factor for LGG. In the univariate Cox regression analysis, the risk score was 1.286 (1.240-1.354) ( $p<0.001$; Figure 3A), with a score of 1.234 (1.176-1.294) in the multivariate Cox regression analysis $(p<0.001$; Figure $3 \mathrm{~B})$, suggesting that NECSig is an independent prognostic predictor. 
Furthermore, the time-dependent ROC curve for the risk score was 0.828 (Figure 3C), which was considerably greater than that for age, sex, radiation, medication, and tumor grade. This result indicates that the risk score based on the NECSig can predict survival in LGG patients.

(A) The univariate and (B) the multivariate Cox regression analyses of relations between clinical factors (including NECSig) and OS. (C) Time-dependent ROC curve analysis for predicting OS after three years based on risk score, age, gender, radiation, pharmaceutical therapies, and tumour grade. AUC: area under the curve.

\section{Correlation between risk score and clinicopathological factors}

We assessed the risk score and the phase between clinical pathological variables to better determine NECSig function in LGG development. There was a different link between risk score and several pathological variables, as indicated in the picture $(p<0.05)$. The risk score of patients older than 45 years was significantly higher than that of patients less than 45 years old ( $p=8.618 \mathrm{e}-06$; Figure $4 \mathrm{~A})$. The risk score of deceased patients was significantly higher than that of surviving patients in the high-risk and low-risk groups ( $p=5.385 \mathrm{e}-07$; Figure 4B). Similarly, Figure $4 \mathrm{C}$ shows that patients with grade III tumours had a considerably higher risk score than patients with grade II tumours $(p=9.512 \mathrm{e}-09)$. It was also found that the risk scores of patients receiving radiotherapy $(p=0.004$; Figure 4D) and pharmaceutical treatments ( $p=0.037$; Figure $4 \mathrm{E}$ ) were higher than those without radiotherapy and pharmaceutical treatments, which may be related to the stage of tumor. This result suggests that patients with severe clinical manifestations may have a higher risk score.

The necroptosis-related mRNA in the cohorts stratified by (A) age, (B) survival state, (C) tumor grade, (D) radiation, and $(\mathrm{E})$ pharmaceutical treatments.

\section{Construction of a predictive nomogram}

The clinically adaptable nomogram incorporating clinicopathological characteristics (age, gender, tumor grade) and the risk score was created in order to estimate the one-, two-, three-, five-, and seven-year survival probabilities in patients with LGG (Figure 5A). The calibration plots the nomogram for one, two, three, five, and seven years suggest that the nomogram estimate of mortality was close to the actual mortality. and that our nomogram was suitable for clinical application (Figure 5B-F). The nomogram may help doctors evaluate the prognostic survival rate of patients and formulate personalized treatment.

(A) Prediction of the one, two-, three-, five-, and seven-year OS of LGG patients with the nomogram.

Nomogram calibration curves for (B) three-year, (C) five-year, and (D) seven-year OS.

\section{Tumor microenvironment (TME) analysis}

Immune cells interact with tumour cells and with each other in the tumour microenvironment. Six algorithms, specifically TIMER, CIBERSORT, quanTIseq, MCP-counter, xCell, and EPIC, were applied to 
estimate the abundance of immune cells infiltrating among high-risk and low-risk groups $(p<0.05$; Figure 6A, Table S3). Comparative analysis of immune cells showed that myeloid dendritic cells (DCs), common lymphoid progenitors, macrophage M2s, macrophages, and neutrophils were the top five different immune cells between the two risk groups. Given the importance of checkpoint-based immunotherapy, we then looked at the differences in immune checkpoint gene expression between the two groups. We found that most immune checkpoints have higher expressions in high-risk patients, such as PDCD-1 (PD-1), CTLA4, and CD274 ( $p<0.05$; Figure 6B).

(A) Immune response heatmaps based on TIMER, CIBERSORT, quanTIseq, MCP-counter, $x$ Cell, and EPIC algorithms for high-risk and low-risk groups. (B) In the TCGA-LGG cohort, immune checkpoint expression differed between the low-risk and high-risk groups. ${ }^{*}, p<0.05 ; * \star, p<0.01 ; * \star *, p<0.001$.

Based on SSGSEA of TCGA-LGG data, a correlation assessment of immune cell subpopulations found that the scores of immune cells, including B cells, CD8+ T cells, DCs, induced DCs (iDCs), macrophages, neutrophils, pDCs, T-helper cells, Th2 cells, TILs, and Tregs were significantly higher in high-risk groups, while the score of NK cells was significantly lower $(p<0.05$; Figure 7A). There were also substantial disparities in immune function scores including APC co-inhibition, APC co-stimulation, CCR, checkpoint, cytolytic activity, HLA, inflammation-promoting, MHC class I, parainflammation, T-cell co-inhibition, T-cell co-stimulation, type-I IFN response, and type-II IFN response between the low-risk and high-risk groups. All immune function scores were higher in the high-risk group ( $p<0.05$; Figure 7B). Six kinds of immune infiltrates in human malignancies have been discovered, ranging from tumour promoting to tumour suppressive. The six types are C1 (wound-healing), C2 (INF-y dominant), C3 (inflammatory), C4 (lymphocyte-depleted), C5 (immunologically-quiet), and C6 (TGF- $\beta$ dominant). Interestingly, the expression of immune cells and immune function is higher in high-risk patients $(p<0.05$; Figure $7 \mathrm{C})$. We also discovered that the immune infiltrates of the high-risk and low-risk groups in the model varied considerably, with immune infiltration type $\mathrm{C} 5$ being the most prominent $(p<0.05$; Figure 7D). In summary, our findings suggest that NECSig is, to some extent, connected to immune cell infiltration.

Differences of (A) immune cells and (B) immune function in high-risk and low-risk groups. (C) Association of four NECSig expressions with different immune infiltrate subtypes tested with ANOVA in TCGA-LGG. (D) Heat map showing the distribution of LGG immune infiltrate subtypes between the lowrisk and high-risk groups. NS, not significant; ${ }^{*}, p<0.05 ; * \star, p<0.01 ; * \star *, p<0.001$. C3: inflammatory, C4: lymphocyte-depleted, C5: immunologically-quiet, and C6: TGF- $\beta$-dominant.

\section{Drug treatment differences in the prognostic model}

Furthermore, we compared the sensitivity of numerous medicines in the high-risk and low-risk categories. The high-risk group had a distinctly lower $\mathrm{IC}_{50}$ value of the drugs including Bortezomib $(p<2.22 \mathrm{e}-16)$, Cyclopamine $(p=4.6 \mathrm{e}-16)$, Dasatinib $(p<2.22 \mathrm{e}-16)$, Rapamycin $(p<2.22 \mathrm{e}-16)$, and Roscovitine $(p=$ $7.4 \mathrm{e}-12$ ) (Figure $8 \mathrm{~A}-\mathrm{E})$, indicating those patients may be more sensitive to these chemotherapy and targeted treatments. We used tumor immune dysfunction and exclusion (TIDE) to evaluate the potential 
clinical efficacy of immunotherapy between the two groups. Since a higher TIDE score represents a higher likelihood of antitumor immune escape, indicating less likely to benefit from immune checkpoint blockade (ICB) treatment. We calculated the TIDE score for each patient, which ranged from -0.5 to 1.23. The TIDE scores of patients in the high-risk group were higher than those in the low-risk group (Figure 8F), indicating the low-risk group could be more sensitive to ICB therapy. Taken together, the NECSig may be a potential predictor of drug sensitivity.

\section{Discussion}

Extracellular stimuli are known to initiate necroptosis and cause inflammation and cell death. When cell apoptosis fails, necroptosis becomes a "failure prevention mechanism" [27]. Recent animal studies have shown that this mechanism can regulate the number of T-cells in peripheral tissues and is also necessary for clearing abnormal lymphocytes during T-cell development [28]. Studies have found that some diseases are also related to the abnormal regulation of necroptosis, such as breast cancer, ovarian cancer, glioma, lung cancer, and leukemia [29-33].

In this study, we constructed four necroptosis-related genes (BID, H2AFY2, MAPK9, and TNFRSF10B) to build a prognostic model. The Kaplan-Meier curve indicates that the high-risk group's survival rate is much lower than that of the low-risk group, and the ROC curve indicates that the model has strong predictive ability. There are also significant differences in clinical characteristics between two groups. In addition, our nomogram showed the potential to improve clinical decision-making and guide the formulation of treatment strategies. A detailed investigation found that the model we created is also related to the type of immune infiltration, high-risk immune checkpoints, and the expression of immune cells and immune functions. Therefore, this research identifies prospective indicators and therapeutic targets of necroptosis signalling pathways.

Four genes in the model are widely involved in tumor progression. BID plays a major role in the process of cell apoptosis and is related to the growth of specific types of gastric cancers [34]. The BID gene is also one of our prognostic model markers [35]. Kun Zhang found that H2AFY2 is a poor prognostic gene in gliomas [36]. MAPK9 is upregulated in normal tissues. In NSCLC tissues, CircRNF20 aggravates the progression of non-small cell lung cancer by activating MAPK9 [37]. YIPF2 can enhance the recirculation of TNFRSF10B to the plasma membrane in non-small cell lung cancer cells, thus promote chemotherapeutic-mediated apoptosis [38]. The reports of these genes in LGG are not complete, and further analysis is needed to explore their effects.

Our study has proved the link between NECSig and the tumor immune microenvironment. It is important to note that the intricate interaction between tumor cells and TME both helps tumor formation and has a substantial influence on immunotherapy, thus affecting OS [39]. The functional enrichment analysis was performed, showing that necroptosis-related genes are mainly involved in immune pathways. Significant differences between high-risk and low-risk groups were found in the immune infiltration analysis, including B-cell, T-cells, CD4, and NK cells, which confirmed the role of NECsig in regulating tumor 
immune infiltration. Tumor immune escape is a phenomena in which tumour cells can avoid detection while attacking the immune system through a number of methods, allowing the tumour cells to live and multiply in the body [40]. TIDE is a creative calculation method used to determine the factors of tumor immune escape [41]. Our results show that low-risk populations may have a more positive response to immunotherapy. In summary, these findings may bring new insights into immunotherapy for the treatment of LGGs.

\section{Conclusion}

In short, we constructed the characteristics of NECSig (including BID, H2AFY2, MAPK9, and TNFRSF10B), and systematically evaluated the correlation between the model, prognosis, and clinicopathological characteristics of LGG patients. Survival analysis and the ROC curve indicate that the feature has good predictive performance. Multivariate Cox and stratified analysis indicate that the four-NECSig feature is an independent prognostic factor of OS. In addition, we built a nomogram that combines the characteristics of NECSig and clinical factors to predict the survival rate of LGG patients. A variety of factors were compared between the high-risk group and the low-risk group, including immune status and response to immunotherapy. This risk profile based on four NECSig may be expected to be utilized for clinical prediction of the prognosis and immunotherapy response of LGG patients.

\section{Materials And Methods}

\section{Identification of Necroptosis-related Differentially Expressed Genes (NECDEs)}

To identify NECDEs, the RNA sequencing (RNA-seq) data and the corresponding clinical features from The Cancer Genome Atlas (TCGA) database (https://portal.gdc.cancer.gov/) was obtained. After data screening and filtering, 529 LGG samples and five normal samples from the TCGA-LGG cohort that had a follow-up time of 30 days or greater were selected. Necroptosis-related genes were collected from the Kyoto Encyclopedia of Genes and Genomes (KEGG) website (https://www.kegg.jp/). The data were download in June 2021. The "limma" package was used to find NECDEs with p-values less than 0.05 .

\section{Function Enrichment Analysis of NECDEs}

Among all the available mRNA expression data for LGG in TCGA, a total of 161 necroptosis-related genes were eventually identified (Table S1). The R packages ggplot2, enrich plot, and cluster profiler packages were used to perform Gene Ontology (GO) enrichment analysis and KEGG pathway analysis.

\section{Development and Validation of the NECSig}

We used univariate Cox regression analysis to find predictive genes. Lasso penalized regression, and multivariate Cox regression. The level of risk was calculated as . Based on their median risk score, patients were divided into high-risk and low-risk categories. To generate Kaplan-Meier survival curves, the R packages "survminer" and "survival" were used. 


\section{Immune Correlation Analysis}

Based on NECSig, we utilized TIMER [21], CIBERSORT [22], quanTIseq [23], MCP-counter [24], xCell [25], and EPIC [26] algorithms are used to analyse the cell types of immune responses in heterogeneous samples to differentiate between high-risk and low-risk groups. The heatmap was used to visualize the differences in the abundance of tumor infiltrating immune cells under distribution algorithms. Immune checkpoints were collected from earlier research. Based on the NECSig, The algorithm was used to calculate the relative concentration of tumor-infiltrating immune cells between the two risk groups based on the NECSig. Finally, the "gsva" programme used ssGSEA to compare immune cells and pathways between the two groups. We analyzed the six types of immune infiltrations using the TCGA dataset and correlated them with the expression levels of four NECSig. Immunotherapeutic response was predicted by TIDE algorithms (http://tide.dfci.harvard.edu/)

\section{The Prediction of Clinical Treatment Response}

The IC50 value determines the sensitivity of certain anticancer medicines. The IC50 values of Bortezomib, Cyclopamine, Dasatinib, Rapamycin, and roscovitine were compared between groups in $\mathrm{R}$ with pRRophetic and ggplot2 using the Wilcoxon signed-rank test.

\section{Statistical Analysis}

$\mathrm{R}$ was used for all statistical analysis (version 4.1.3). To explore the link between the prognostic model risk score and stemness, stromal score, immunological score, and drug sensitivity, the R programme "limma" was employed. To investigate differences across groups, the Kruskal-Wallis test was utilized. The nomogram was created with the R software "rms". Clinical data was analysed using the chi-squared test. The Student's t-test was performed to determine the statistical significance of the difference between the two groups, with $p<0.05$ indicating that the difference was statistically significant.

\section{Declarations}

\section{Ethics approval and consent to participate}

Not applicable

\section{Consent for publication}

Not applicable

\section{Availability of data and materials}

All data generated or analyzed during this study are included in this published article.

\section{Competing interests}


The authors declare that they have no competing interests.

\section{Funding}

Not applicable

\section{Authors' contributions}

Conception and design: KZ, ZC. Administrative support: ZC. Provision of study materials: KZ, GZ, ZC. Collection and assembly of data: GZ, WH, JZ. Data analysis and interpretation: KZ, GZ, WH, JZ. Manuscript writing: All authors. Final approval of manuscript: All authors.

\section{Acknowledgements}

Not applicable

\section{References}

[1] Louis DN, Perry A, Reifenberger G, von Deimling A, Figarella-Branger D, Cavenee WK, et al. The 2016 World Health Organization Classification of Tumors of the Central Nervous System: a summary. Acta Neuropathol (2016) 131(6):803-20. doi: 10.1007/s00401-016-1545-1

[2] Ostrom QT, Patil N, Cioffi G, Waite K, Kruchko C, Barnholtz-Sloan JS. CBTRUS Statistical Report: Primary Brain and Other Central Nervous System Tumors Diagnosed in the United States in 2013-2017. Neuro Oncol (2020) 22(12 Suppl 2):iv1-iv96. doi: 10.1093/neuonc/noaa200

[3] Claus EB, Black PM. Survival rates and patterns of care for patients diagnosed with supratentorial lowgrade gliomas: data from the SEER program, 1973-2001. Cancer (2006) 106(6):1358-63. doi: 10.1002/cncr.21733

[4] Claus EB, Walsh KM, Wiencke JK, Molinaro AM, Wiemels JL, Schildkraut JM, et al. Survival and lowgrade glioma: the emergence of genetic information. Neurosurg Focus (2015) 38(1):E6. doi: 10.3171/2014.10.FOCUS12367

[5] von Mässenhausen A, Tonnus W, Himmerkus N, Parmentier S, Saleh D, Rodriguez D, et al. Phenytoin inhibits necroptosis. Cell Death Dis (2018) 9(3):359. doi: 10.1038/s41419-018-0394-3

[6] Siegmund D, Ehrenschwender M, Wajant H. TNFR2 unlocks a RIPK1 kinase activity-dependent mode of proinflammatory TNFR1 signaling. Cell Death Dis (2018) 9(9):921. doi: 10.1038/s41419-018-0973-3

[7] Snyder AG, Hubbard NW, Messmer MN, Kofman SB, Hagan CE, Orozco SL, et al. Intratumoral activation of the necroptotic pathway components RIPK1 and RIPK3 potentiates antitumor immunity. Sci Immunol (2019) 4(36):eaaw2004. doi: 10.1126/sciimmunol.aaw2004 
[8] Samson AL, Zhang Y, Geoghegan ND, Gavin XJ, Davies KA, Mlodzianoski MJ, et al. MLKL trafficking and accumulation at the plasma membrane control the kinetics and threshold for necroptosis. Nat Commun (2020) 11(1):3151. doi: 10.1038/s41467-020-16887-1

[9] Yoon S, Kovalenko A, Bogdanov K, Wallach D. MLKL, the Protein that Mediates Necroptosis, Also Regulates Endosomal Trafficking and Extracellular Vesicle Generation. Immunity (2017) 47(1):51-65.e7. doi: 10.1016/j.immuni.2017.06.001

[10] Dovey CM, Diep J, Clarke BP, Hale AT, McNamara DE, Guo H, et al. MLKL Requires the Inositol Phosphate Code to Execute Necroptosis. Mol Cell (2018) 70(5):936-948.e7. doi:

10.1016/j.molcel.2018.05.010

[11] Dondelinger Y, Darding M, Bertrand MJ, Walczak H. Poly-ubiquitination in TNFR1-mediated necroptosis. Cell Mol Life Sci (2016) 73(11-12):2165-76. doi: 10.1007/s00018-016-2191-4 [12] Chen AQ, Fang Z, Chen XL, Yang S, Zhou YF, Mao L, et al. Microglia-derived TNF-a mediates endothelial necroptosis aggravating blood brain-barrier disruption after ischemic stroke. Cell Death Dis (2019) 10(7):487. doi: $10.1038 / \mathrm{s} 41419-019-1716-9$

[13] Li X, Zhang M, Huang X, Liang W, Li G, Lu X, et al. Ubiquitination of RIPK1 regulates its activation mediated by TNFR1 and TLRs signaling in distinct manners. Nat Commun (2020) 11(1):6364. doi: 10.1038/s41467-020-19935-y

[14] Newton K, Wickliffe KE, Dugger DL, Maltzman A, Roose-Girma M, Dohse M, et al. Cleavage of RIPK1 by caspase-8 is crucial for limiting apoptosis and necroptosis. Nature (2019) 574(7778):428-431. doi: $10.1038 / \mathrm{s} 41586-019-1548-\mathrm{x}$

[15] Lalaoui N, Boyden SE, Oda H, Wood GM, Stone DL, Chau D, et al. Mutations that prevent caspase cleavage of RIPK1 cause autoinflammatory disease. Nature (2020) 577(7788):103-108. doi: $10.1038 / \mathrm{s} 41586-019-1828-5$

[16] Tummers B, Mari L, Guy CS, Heckmann BL, Rodriguez DA, Rühl S, et al. Caspase-8-Dependent Inflammatory Responses Are Controlled by Its Adaptor, FADD, and Necroptosis. Immunity (2020) 52(6):994-1006.e8. doi: 10.1016/j.immuni.2020.04.010

[17] Someda M, Kuroki S, Miyachi H, Tachibana M, Yonehara S. Caspase-8, receptor-interacting protein kinase 1 (RIPK1), and RIPK3 regulate retinoic acid-induced cell differentiation and necroptosis. Cell Death Differ (2020) 27(5):1539-1553. doi: 10.1038/s41418-019-0434-2

[18] Zhou J, Li G, Han G, Feng S, Liu Y, Chen J, et al. Emodin induced necroptosis in the glioma cell line U251 via the TNF-a/RIP1/RIP3 pathway. Invest New Drugs (2020) 38(1):50-59. doi: 10.1007/s10637-01900764-w 
[19] Huangfu M, Wei R, Wang J, Qin J, Yu D, Guan X, et al. Osthole induces necroptosis via ROS overproduction in glioma cells. FEBS Open Bio (2021) 11(2):456-467. doi: 10.1002/2211-5463.13069 [20] LingHu HR, Luo H, Gang L. Bufalin Induces Glioma Cell Death by Apoptosis or Necroptosis. Onco Targets Ther (2020) 13:4767-4778. doi: 10.2147/OTT.S242567

[21] Li T, Fan J, Wang B, Traugh N, Chen Q, Liu JS, et al. TIMER: A Web Server for Comprehensive Analysis of Tumor-Infiltrating Immune Cells. Cancer Res (2017) 77(21):e108-e110. doi: 10.1158/0008-5472.CAN17-0307

[22] Charoentong P, Finotello F, Angelova M, Mayer C, Efremova M, Rieder D, et al. Pan-cancer Immunogenomic Analyses Reveal Genotype-Immunophenotype Relationships and Predictors of Response to Checkpoint Blockade. Cell Rep (2017) 18(1):248-262. doi: 10.1016/j.celrep.2016.12.019

[23] Finotello F, Mayer C, Plattner C, Laschober G, Rieder D, Hackl H, et al. Molecular and pharmacological modulators of the tumor immune contexture revealed by deconvolution of RNA-seq data. Genome Med (2019) 11(1):34. doi: 10.1186/s13073-019-0638-6

[24] Shi J, Jiang D, Yang S, Zhang X, Wang J, Liu Y, et al. LPAR1, Correlated With Immune Infiltrates, Is a Potential Prognostic Biomarker in Prostate Cancer. Front Oncol (2020) 10:846. doi: 10.3389/fonc. 2020.00846

[25] Aran D, Hu Z, Butte AJ. xCell: digitally portraying the tissue cellular heterogeneity landscape. Genome Biol (2017) 18(1):220. doi: 10.1186/s13059-017-1349-1

[26] Racle J, de Jonge K, Baumgaertner P, Speiser DE, Gfeller D. Simultaneous enumeration of cancer and immune cell types from bulk tumor gene expression data. Elife (2017) 6:e26476. doi:

10.7554/eLife.26476

[27] Zhou W, Yuan J. SnapShot: Necroptosis. Cell (2014) 158(2):464-464.e1. doi:

10.1016/j.cell.2014.06.041

[28] Newton K, Manning G. Necroptosis and Inflammation. Annu Rev Biochem (2015) 85:743-63. doi: 10.1146/annurev-biochem-060815-014830

[29] Shen F, Pan X, Li M, Chen Y, Jiang Y, He J. Pharmacological Inhibition of Necroptosis Promotes Human Breast Cancer Cell Proliferation and Metastasis. Onco Targets Ther (2020) 13:3165-3176. doi: $10.2147 /$ OTT.S246899

[30] Liu L, Fan J, Ai G, Liu J, Luo N, Li C, et al. Berberine in combination with cisplatin induces necroptosis and apoptosis in ovarian cancer cells. Biol Res (2019) 52(1):37. doi: 10.1186/s40659-019-0243-6

[31] Lu B, Gong X, Wang ZQ, Ding Y, Wang C, Luo TF, et al. Shikonin induces glioma cell necroptosis in vitro by ROS overproduction and promoting RIP1/RIP3 necrosome formation. Acta Pharmacol Sin (2017) 38(11):1543-1553. doi: 10.1038/aps.2017.112

Page 12/21 
[32] Liu X, Zhang Y, Gao H, Hou Y, Lu JJ, Feng Y, et al. Induction of an MLKL mediated non-canonical necroptosis through reactive oxygen species by tanshinol A in lung cancer cells. Biochem Pharmacol (2020) 171:113684. doi: 10.1016/j.bcp.2019.113684

[33] Huang X, Xiao F, Li Y, Qian W, Ding W, Ye X. Bypassing drug resistance by triggering necroptosis: recent advances in mechanisms and its therapeutic exploitation in leukemia. J Exp Clin Cancer Res (2018) 37(1):310. doi: 10.1186/s13046-018-0976-z

[34] Gryko M, Pryczynicz A, Zareba K, Kędra B, Kemona A, Guzińska-Ustymowicz K. The expression of Bcl2 and BID in gastric cancer cells. J Immunol Res (2014) 2014:953203. doi: 10.1155/2014/953203

[35] Xu Y, Li R, Li X, Dong N, Wu D, Hou L, et al. An Autophagy-Related Gene Signature Associated With Clinical Prognosis and Immune Microenvironment in Gliomas. Front Oncol (2020) 10:571189. doi: $10.3389 /$ fonc. 2020.571189

[36] Zhang K, Zhao H, Zhang K, Hua C, Qin X, Xu S. Chromatin-regulating genes are associated with postoperative prognosis and isocitrate dehydrogenase mutation in astrocytoma. Ann Transl Med (2020) 8(23):1594. doi: 10.21037/atm-20-7229

[37] Wang ZX, Zhao Y, Wang YB, Zhang Q, Zou QX, Liang FH, et al. CircRNF20 aggravates the progression of non-small-cell lung carcinoma by activating MAPK9. Eur Rev Med Pharmacol Sci (2020) 24(19):99819989. doi: 10.26355/eurrev_202010_23211

[38] Wang Y, Guo S, Li D, Tang Y, Li L, Su L, et al. YIPF2 promotes chemotherapeutic agent-mediated apoptosis via enhancing TNFRSF10B recycling to plasma membrane in non-small cell lung cancer cells. Cell Death Dis (2020) 11(4):242. doi: 10.1038/s41419-020-2436-x

[39] Lei X, Lei Y, Li JK, Du WX, Li RG, Yang J, et al. Immune cells within the tumor microenvironment: Biological functions and roles in cancer immunotherapy. Cancer Lett (2020) 470:126-133. doi: 10.1016/j.canlet.2019.11.009

[40] Khong HT, Restifo NP. Natural selection of tumor variants in the generation of "tumor escape" phenotypes. Nat Immunol (2002) 3(11):999-1005. doi: 10.1038/ni1102-999

[41] Jiang P, Gu S, Pan D, Fu J, Sahu A, Hu X, et al. Signatures of T cell dysfunction and exclusion predict cancer immunotherapy response. Nat Med (2018) 24(10):1550-1558. doi: 10.1038/s41591-018-0136-1

\section{Figures}


A

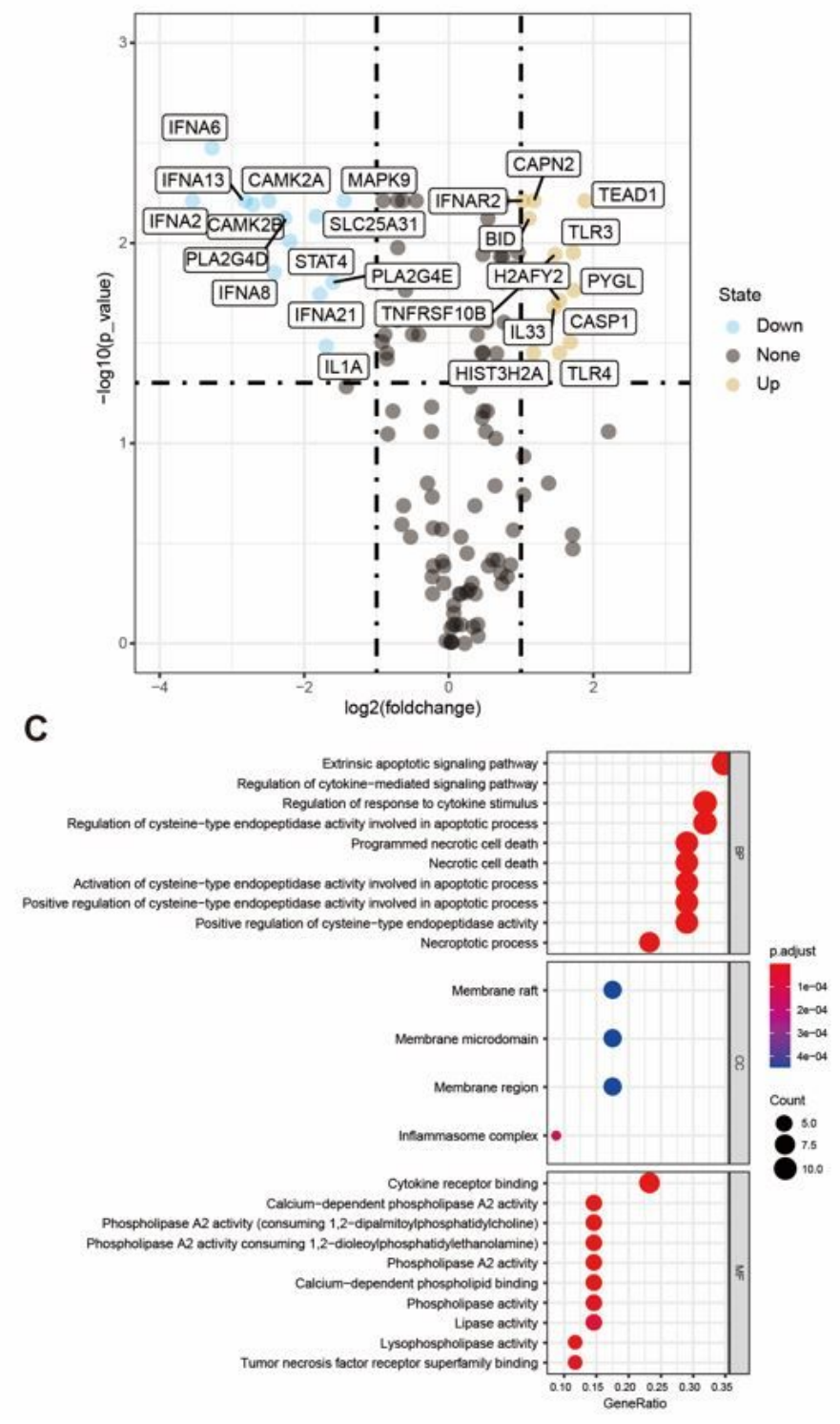

B

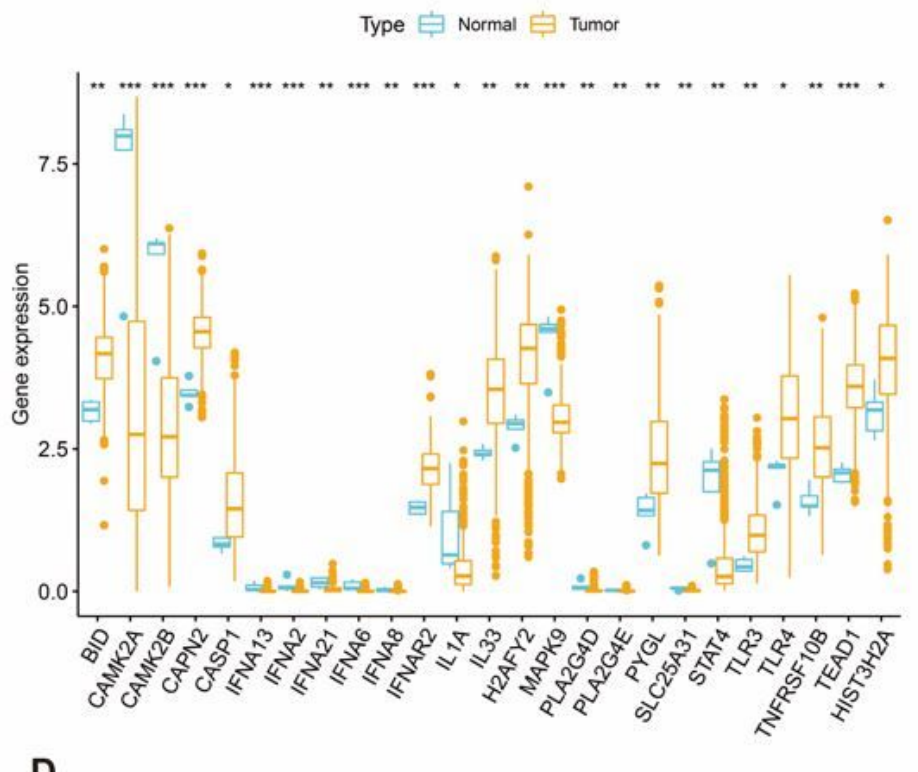

D

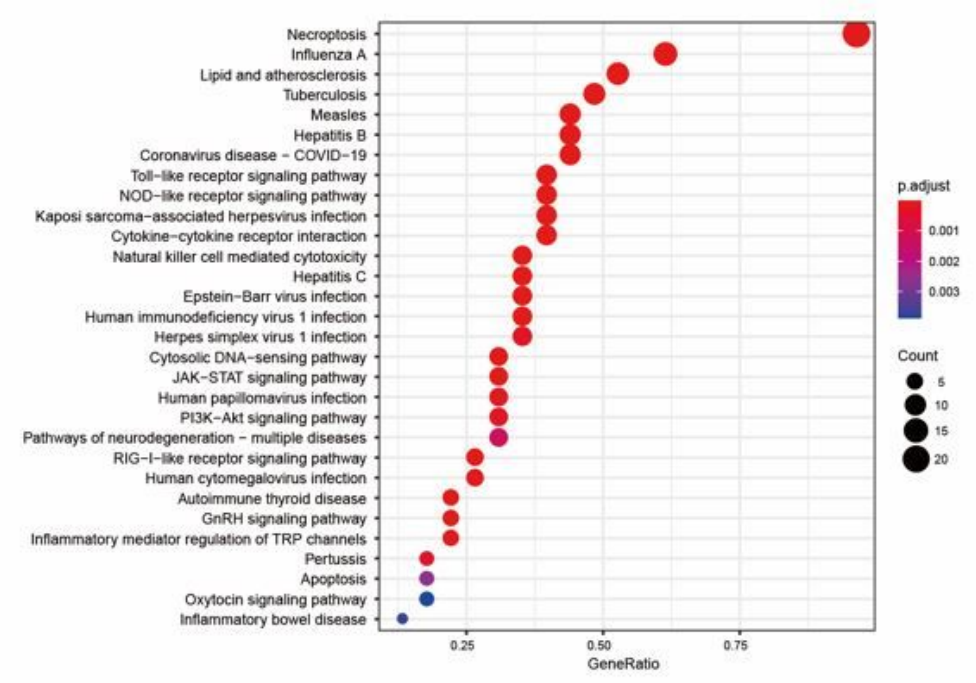

\section{Figure 1}

Identification of NECDEs and functional enrichment (A) The NECDEs between LGG patients and normal controls. (B) The expression of different NECDEs in the normal group and tumor group. (C) The GO function enrichment analysis, including the top 10 pathways corresponding to biological processes, cellular components, and molecular functions. (D) The top 30 pathways in the KEGG functional enrichment analysis. *, $p<0.05 ; * \star, p<0.01 ; * \star *, p<0.001$. 
A pvalue Hazard ratio

BID

CASP1 $\quad<0001 \quad 1215(1.155-1.279)$

IFNAR2 $<0.001 \quad 1.203(1.088-1.329)$

IL.1A $\quad<0.001 \quad 1.458(1.172-1.813)$

H2AFY2 $<0.001 \quad 0.937(0.915-0.959)$

MAPK9 $\quad 0.004 \quad 0.899(0.837-0.966)$

PYGL $\quad<0.001 \quad 1.073(1.052-1.095)$

TLR3 $\quad<0.001 \quad 1.530(1.345-1.740)$

HIST3H2A $<0.001 \quad 0.958(0.938-0.978)$

E

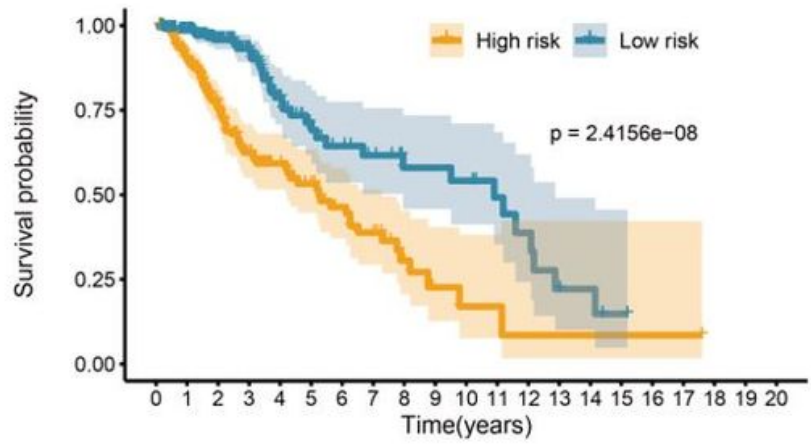

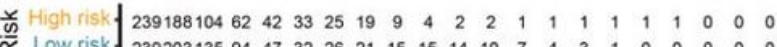

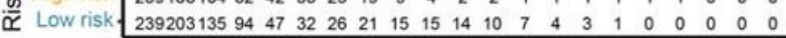
$\begin{array}{lllllllllllllllllllll}0 & 1 & 2 & 3 & 4 & 5 & 6 & 7 & 8 & 9 & 10 & 11 & 12 & 13 & 14 & 15 & 16 & 17 & 18 & 19 & 20\end{array}$ Time(years)

B
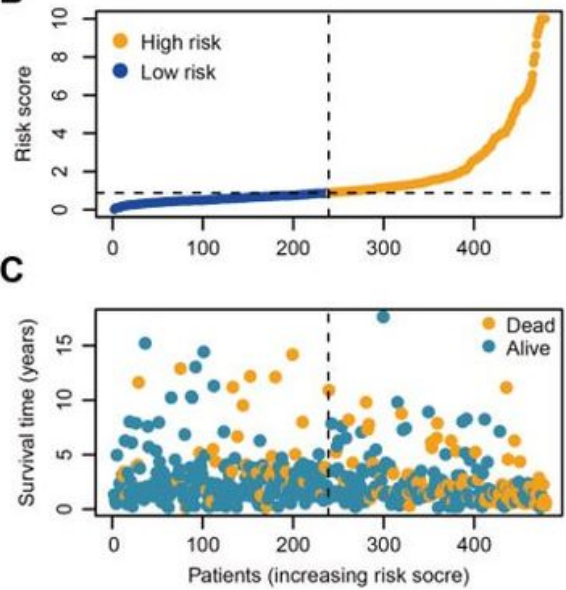

$\mathbf{F}$

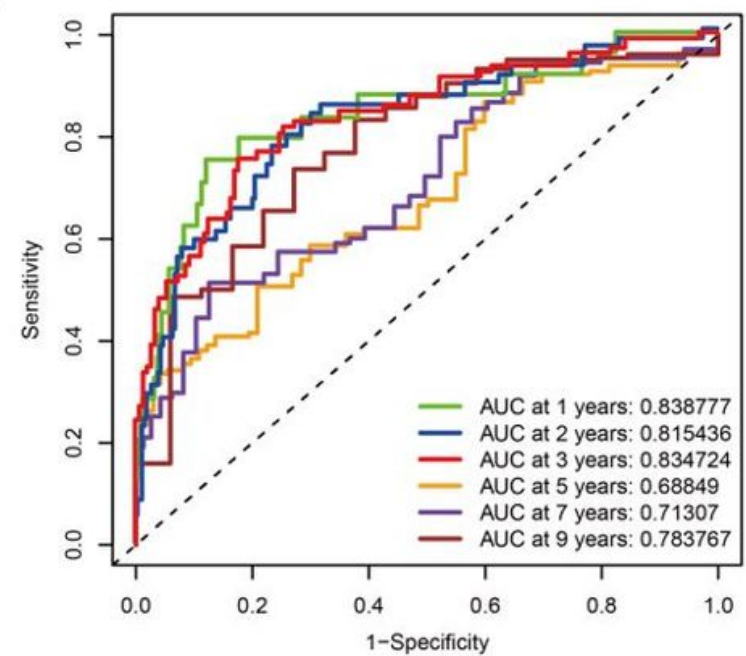

\section{Figure 2}

Development of a risk score model based on the NECSig in LGG (A) The prognostic value of the 10 chosen NECDEs. (B) The risk curve is determined by the risk score for each sample. (C) The patient survival status of two groups. Blue and orange scatters indicate alive and dead respectively. (D) The distribution of four NECSig genes in the high-risk and low-risk groups based on the TCGA-LGG cohort. (E) Kaplan-Meier curves for patients in the high-risk and low-risk groups overall survival. (F) ROC curves of the risk score predicting one-, two-, three-, five-, seven-, and nine-year survival based on the TCGA-LGG cohort. AUC: area under the curve. 
A

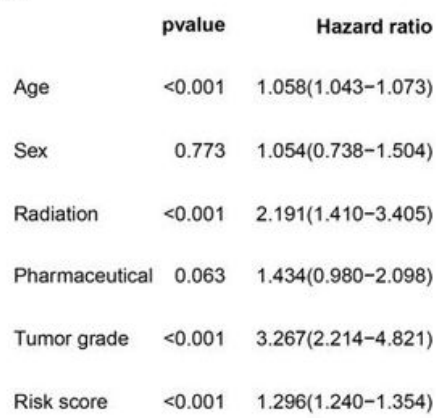

B

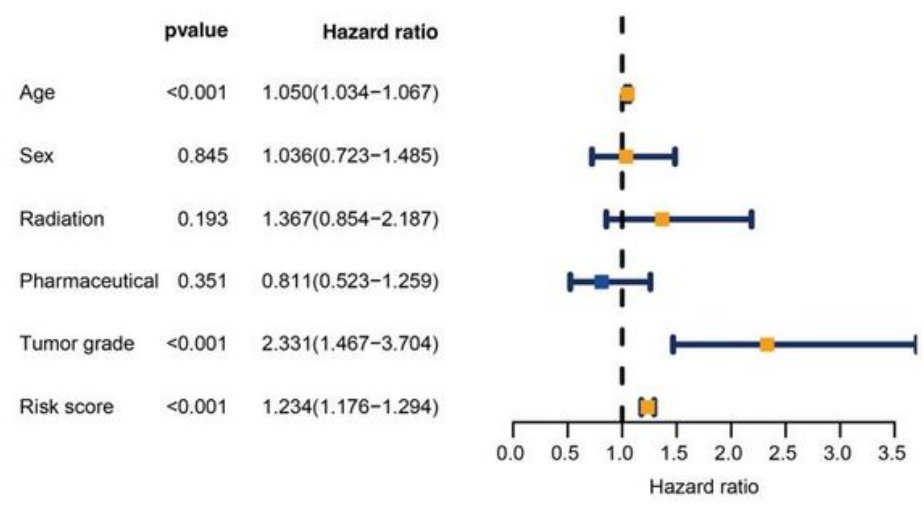

C

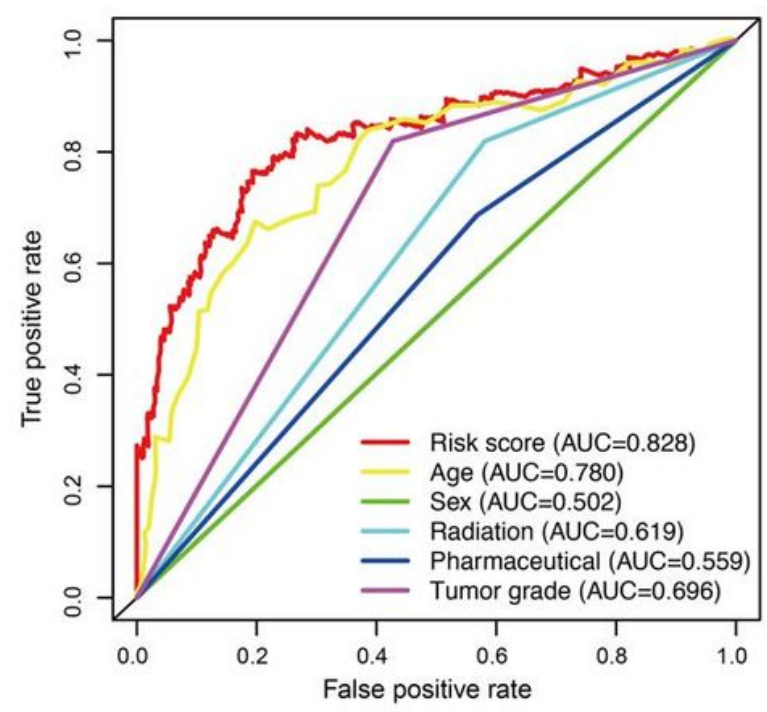

\section{Figure 3}

Evaluation of NECSig as an independent prognostic factor for LGG (A) The univariate and (B) the multivariate Cox regression analyses of relations between clinical factors (including NECSig) and OS. (C) Time-dependent ROC curve analysis for predicting OS after three years based on risk score, age, gender, radiation, pharmaceutical therapies, and tumour grade. AUC: area under the curve. 
A
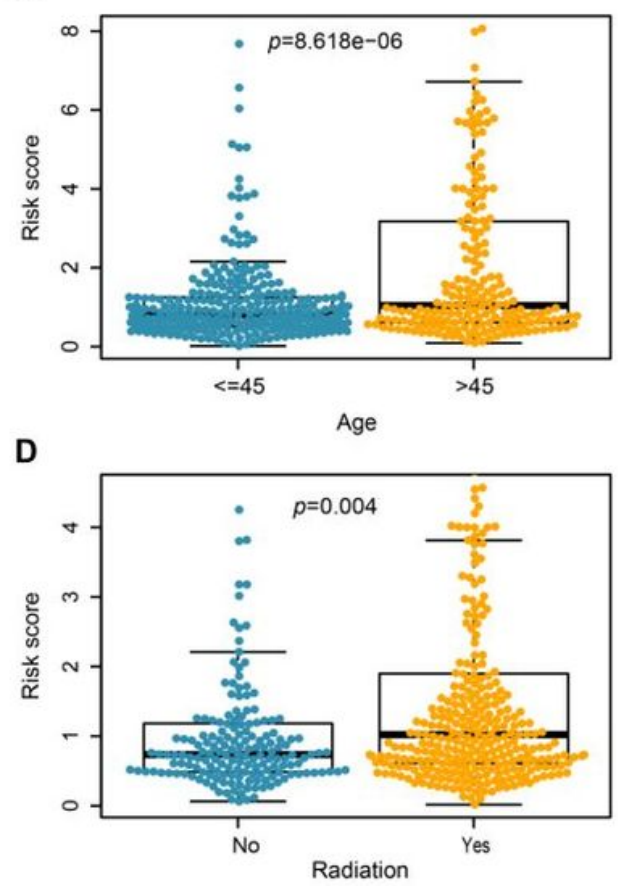

B

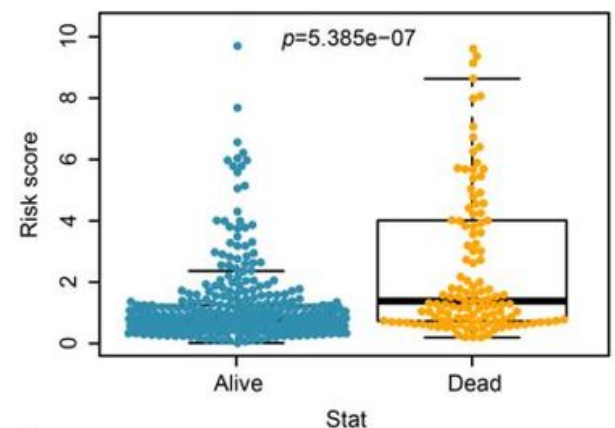

E

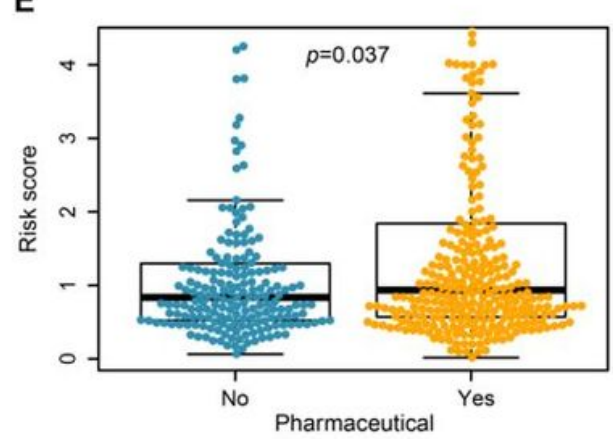

C

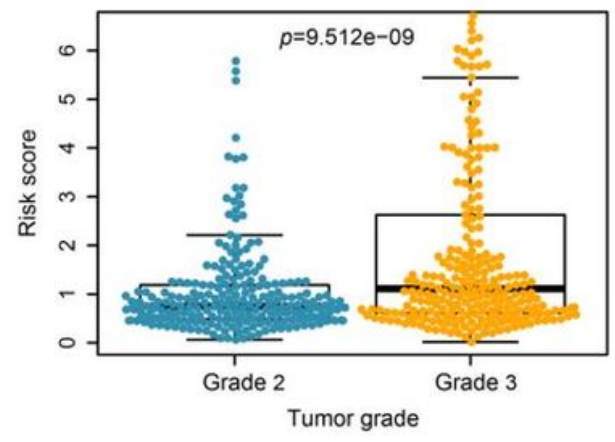

\section{Figure 4}

Correlation between risk score and clinicopathological factors The necroptosis-related mRNA in the cohorts stratified by $(A)$ age, $(B)$ survival state, $(C)$ tumor grade, (D) radiation, and (E) pharmaceutical treatments. 
A
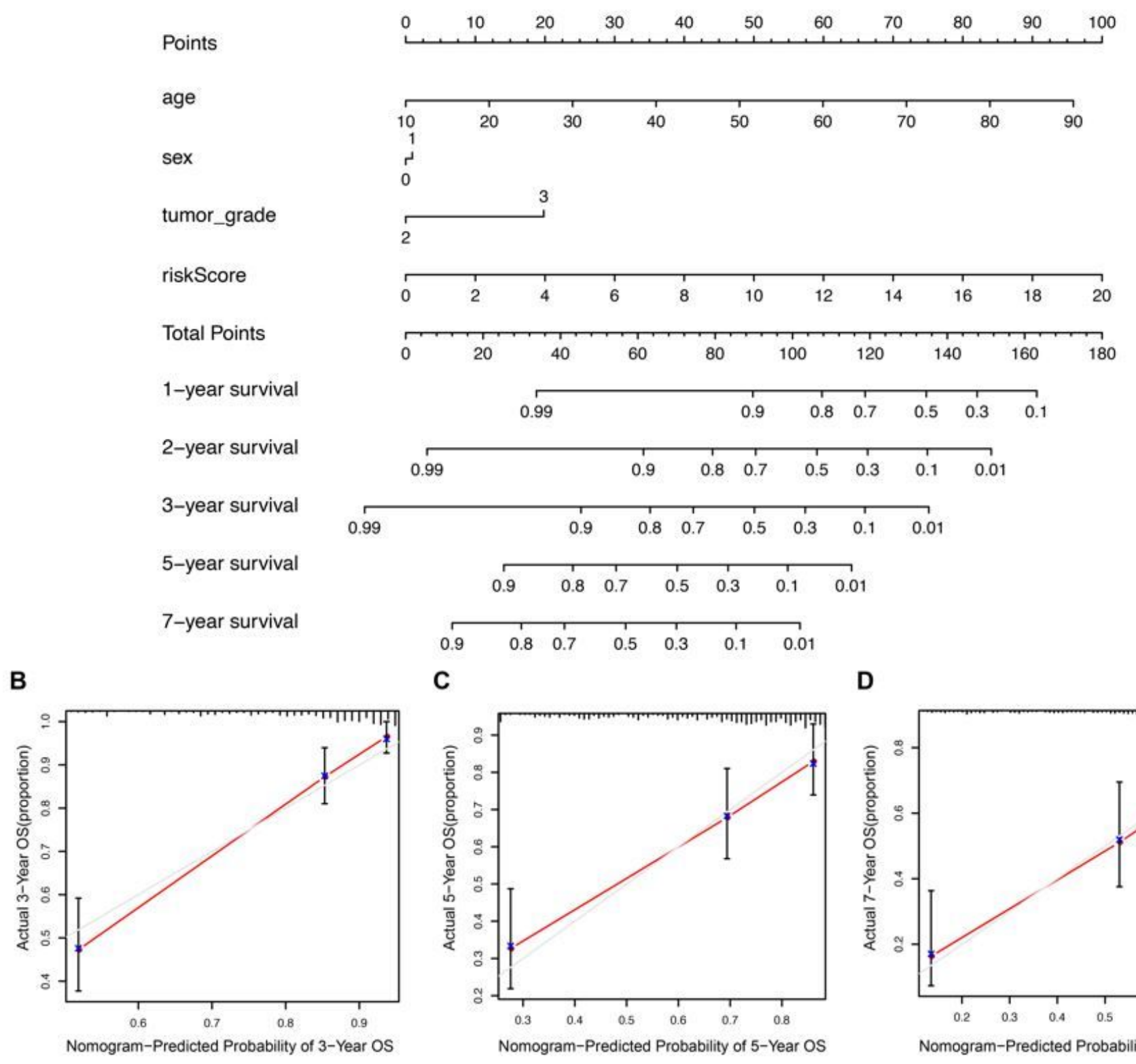

C

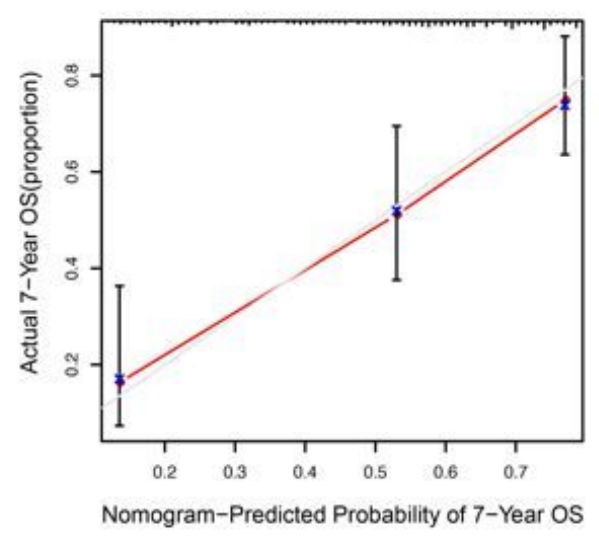

Figure 5

Prognostic nomogram for predicting survival (A) Prediction of the one-, two-, three-, five-, and seven-year OS of LGG patients with the nomogram. 
A

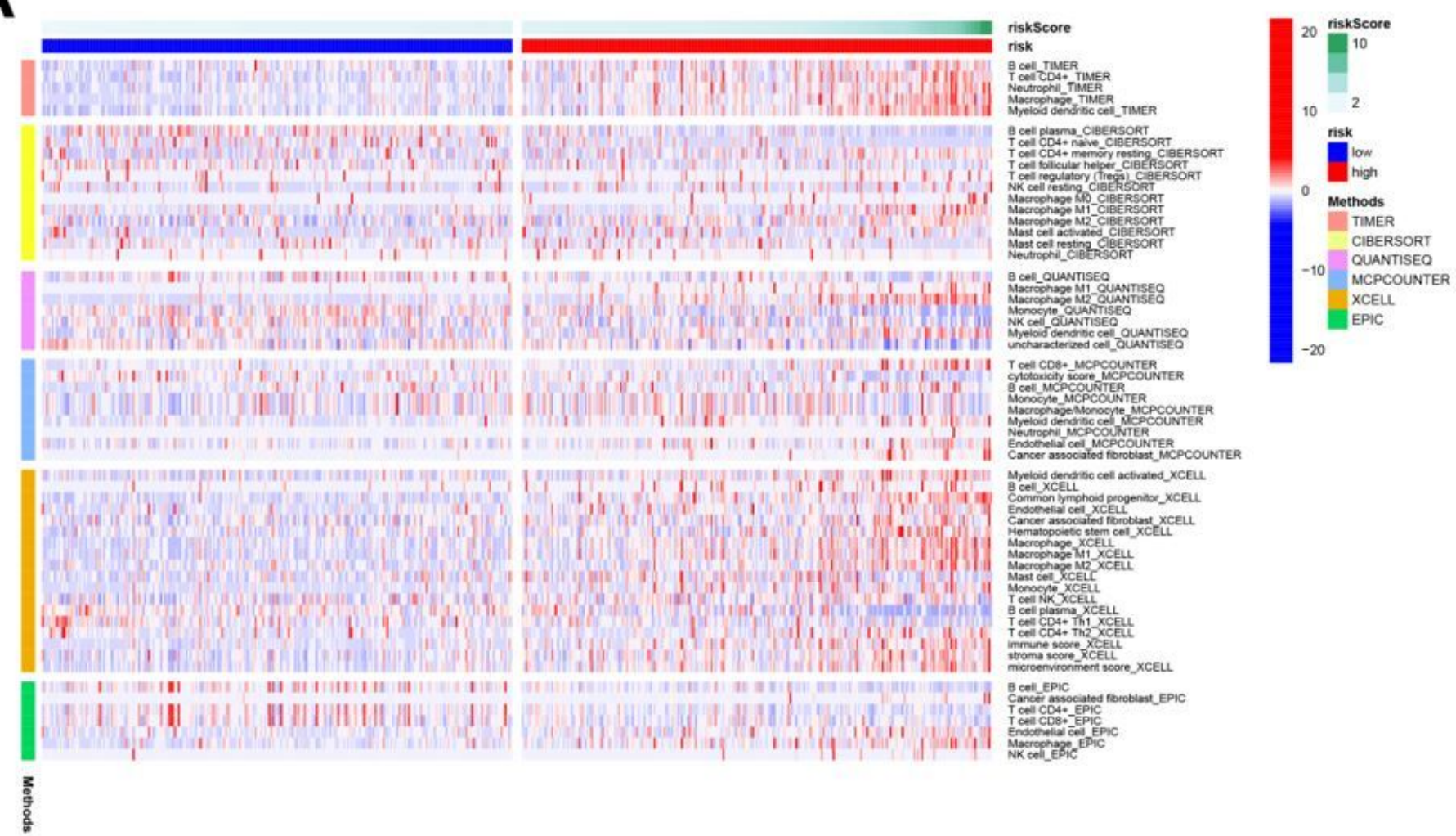

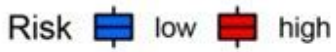

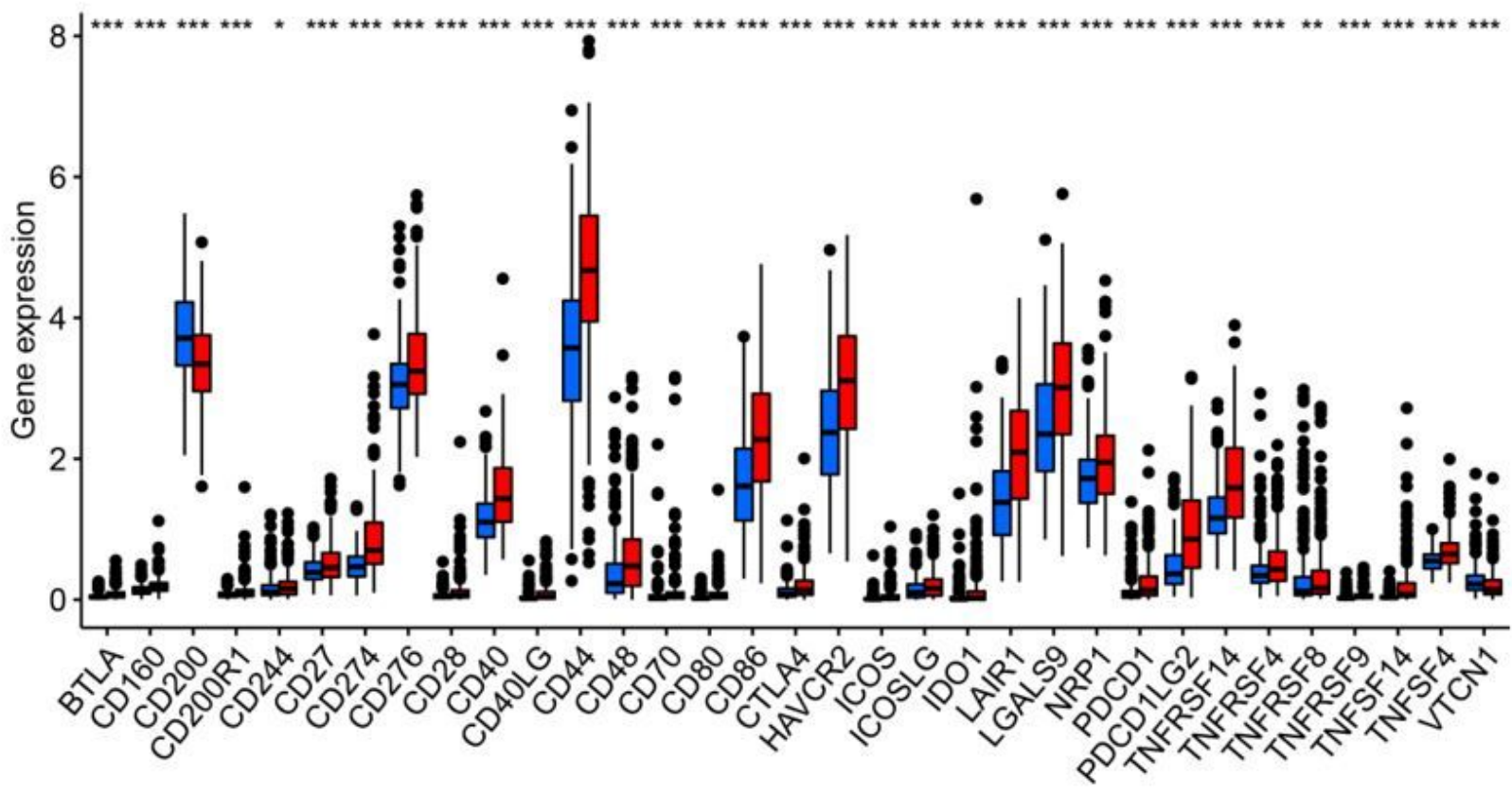

Figure 6

Immune infiltration and immune checkpoint analysis (A) Immune response heatmaps based on TIMER, CIBERSORT, quanTIseq, MCP-counter, $x$ Cell, and EPIC algorithms for high-risk and low-risk groups. (B) In the TCGA-LGG cohort, immune checkpoint expression differed between the low-risk and high-risk groups. $*, p<0.05 ; * \star, p<0.01 ; * \star *, p<0.001$. 
A

Risk 由 low 户 high
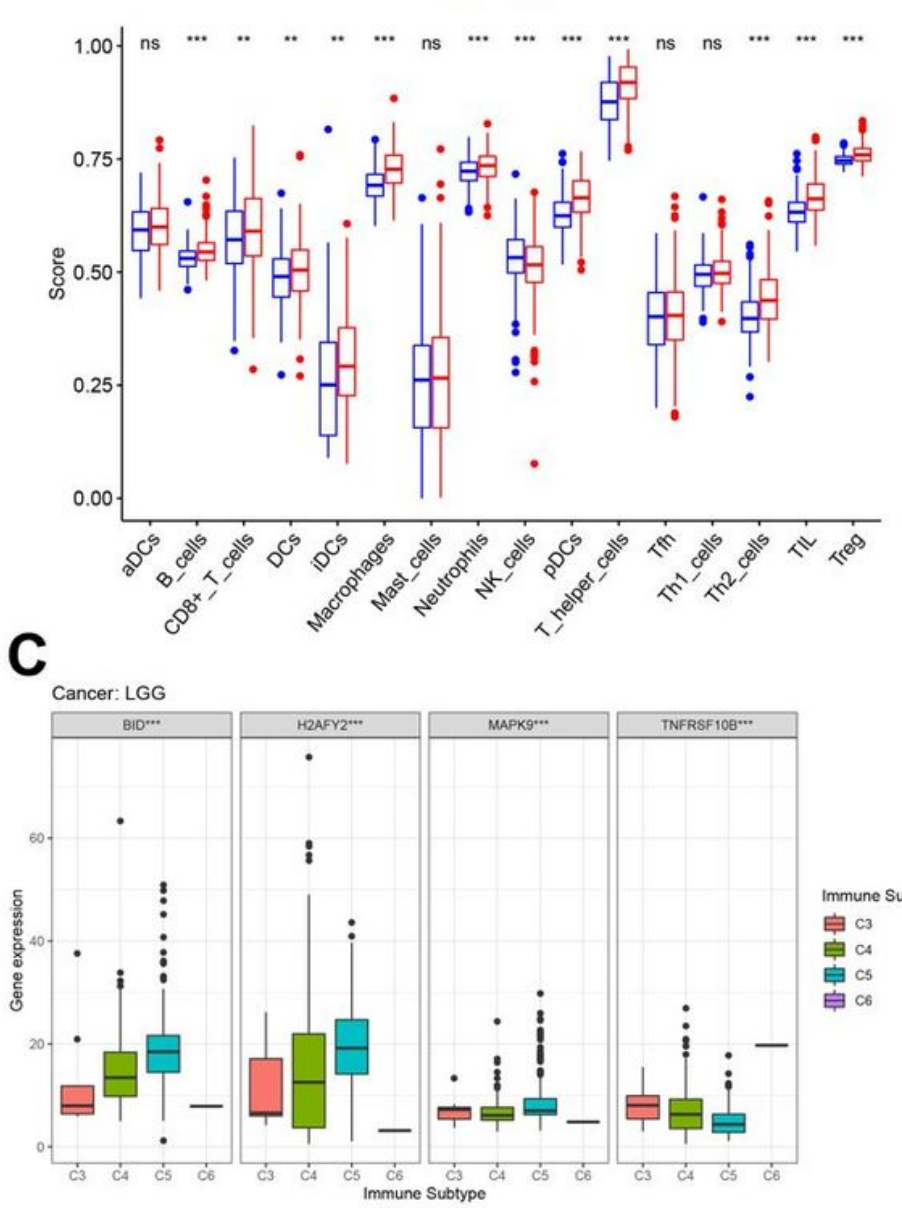

B
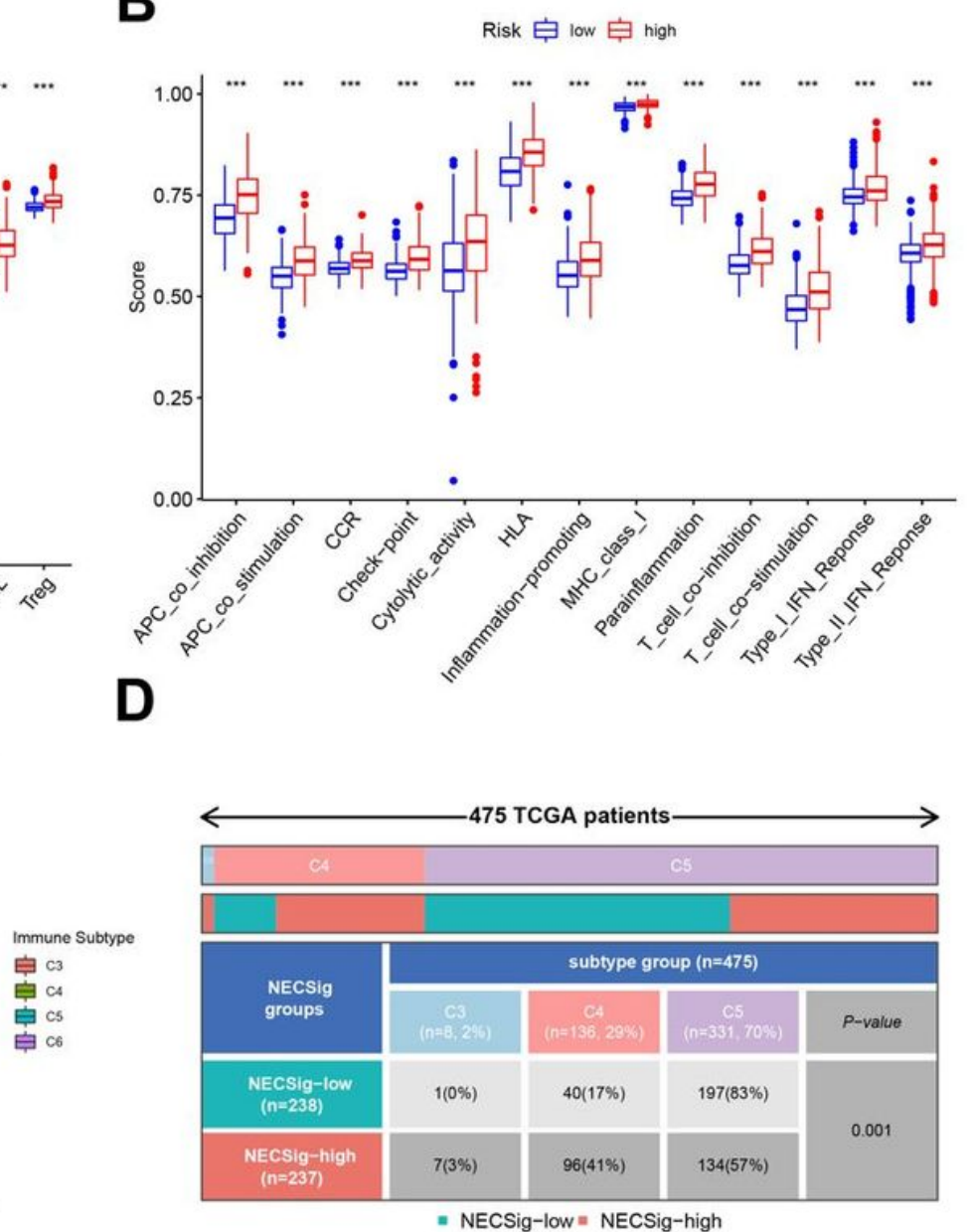

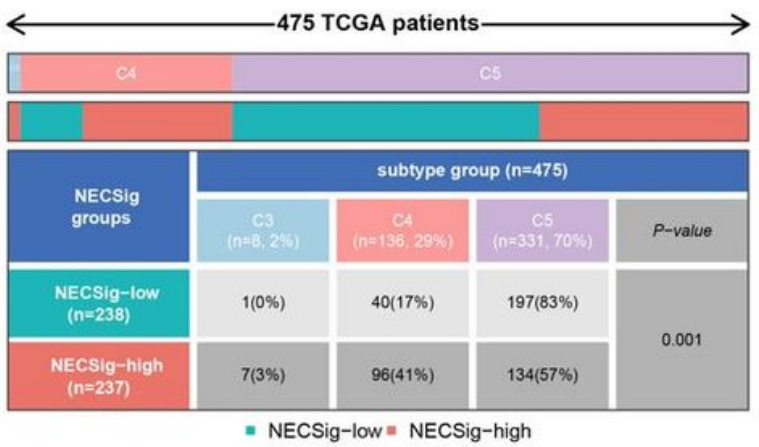

Figure 7

Comparison of the immunity analysis between different risk groups Differences of $(A)$ immune cells and (B) immune function in high-risk and low-risk groups. (C) Association of four NECSig expressions with different immune infiltrate subtypes tested with ANOVA in TCGA-LGG. (D) Heat map showing the distribution of LGG immune infiltrate subtypes between the low-risk and high-risk groups. NS, not significant; *, $\mathrm{p}<0.05$; **, $\mathrm{p}<0.01$; ***, $\mathrm{p}<0.001$. C3: inflammatory, C4: lymphocyte-depleted, C5: immunologically-quiet, and C6: TGF- $\beta$-dominant. 
A

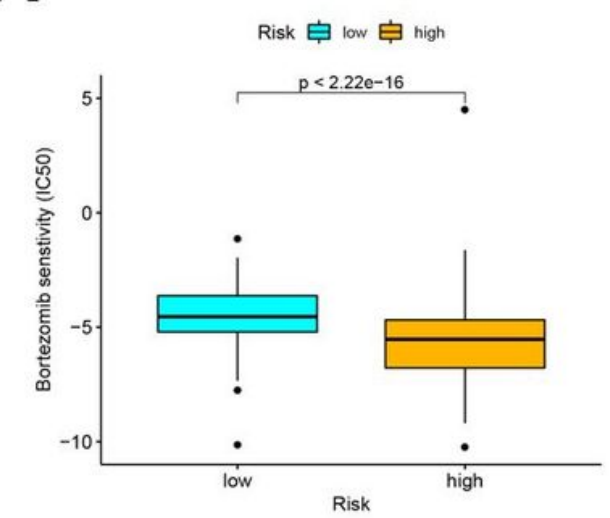

D

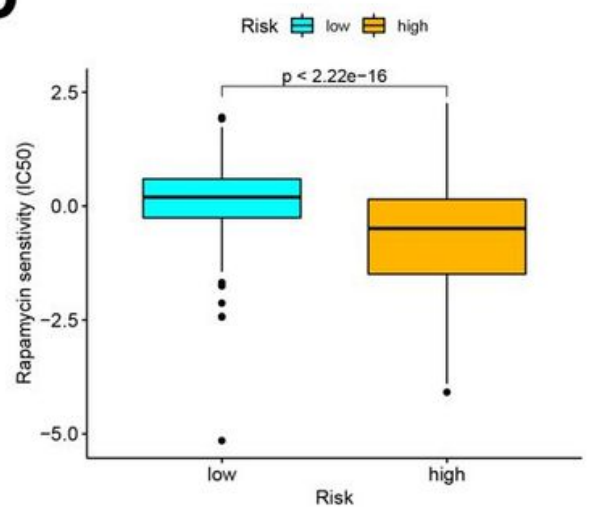

B

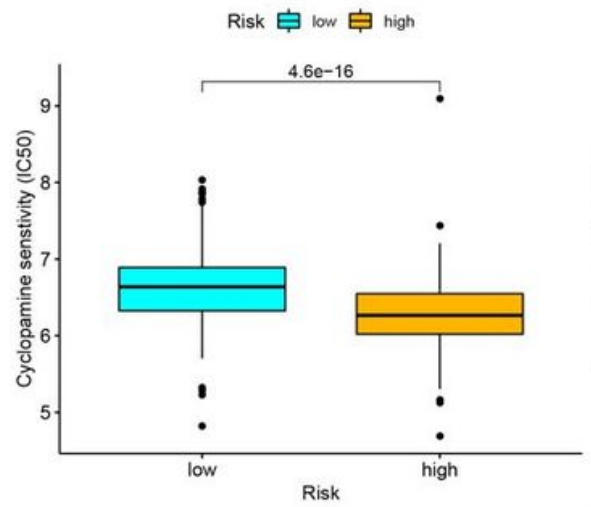

E

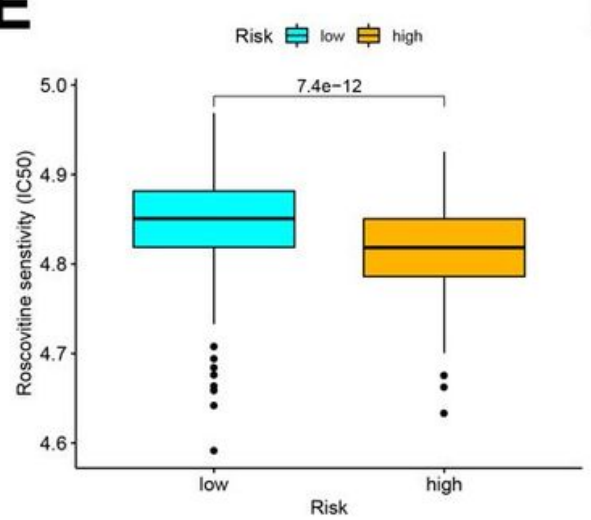

C

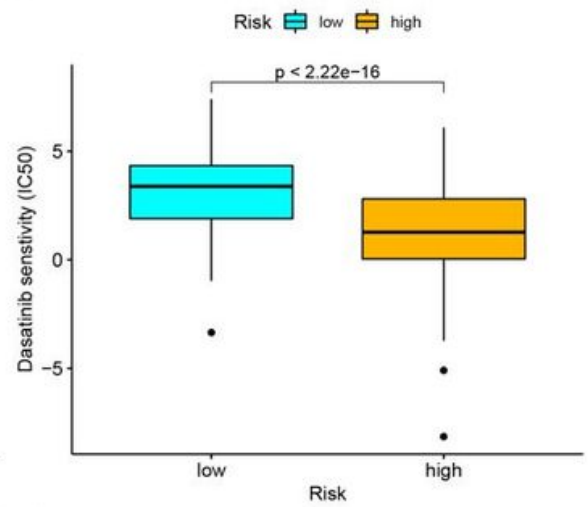

$\mathbf{F}$

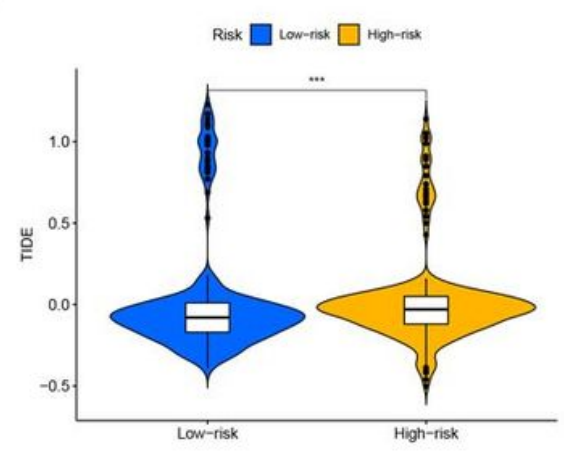

Figure 8

Comparison of responses to medical treatment between the high-risk and low-risk groups The high-risk scores were related to lower IC50 values for chemotherapeutics and target-directed treatments, such as (A) Bortezomib, (B) Cyclopamine, (C) Dasatinib, (D) Rapamycin, and (E) Roscovitine. (F) TIDE score in low-risk and high-risk groups in the cohort from TCGA-LGG. ${ }^{* \star}, \mathrm{p}<0.001$.

\section{Supplementary Files}

This is a list of supplementary files associated with this preprint. Click to download.

- TableS1Listofnecroptosisrelatedgenes.xlsx

- TableS3Theabundanceofimmunecellsinfiltratingamonghighriskandlowriskgroups.xlsx

- TableS2Differentialanalysisinnecroptosisrelatedgenes.xlsx 